\title{
CORNEA MODEL: Light-Scattering Patterns from a Two-Dimensional Nonrandom Assembly of Anisotropic Rods
}

\author{
Mahadevappa Kumbar and Frederick A. Bettelheim \\ Department of Chemistry, Adelphi University, \\ Garden City, New York 11530, U.S.A.
}

(Received December 11, 1974)

\begin{abstract}
The light scattered by a cornea occurs mainly at small angles. The patterns obtained experimentally showed a characteristic maxima at $0^{\circ}$ angles and a four lobe $0-90^{\circ}$ pattern in the $I_{+}$mode and a two lobe $0-180^{\circ}$ pattern in the $I_{\|}$mode. A model system is presented here in which anisotropic rods (collagen fibrils) embedded in an isotropic medium (ground substances) form a two-dimensional assembly (lamellae) which are stacked in a nonrandom fashion (stroma). The light-scattering patterns from such a model system were calculated by varying a number of parameters, such as the length of the rod, its average orientation, etc. Thus the role of each parameter in the model in influencing light-scattering patterns in $I_{+}$and $I_{\|}$mode was evaluated. Finally, the computer-simulated patterns were matched to the experimental patterns and the numerical values of the parmeters of the model were obtained.
\end{abstract}

KEY WORDS Light Scattering / Anisotropic Rods / Nonrandom

Distribution / Bovine Cornea / Collagen Fibers /

The cornea of the eye is the only tissue with high collagen content $(15 \%)$ that is transparent. It is composed of five layers lying parallel to the surfaces. These layers are, proceeding from anterior to posterior: epithelium, basement membrane, stroma, Descemet membrane, and endothelium. Of these, the stroma occupies about $9 / 10$ of the total thickness. ${ }^{1}$

The stroma contains a few hundreds of lamellae, depending on the species. These lamellae are sheets in which collagen fibrils run parallel to each other. The collagen fibrils are surrounded by a ground substance of proteoglycans, which have a heterogenous distribution of keratan sulfate $(2 / 3)$ and chondroitin-4-sulfate (1/3) chains on protein cores. The proteoglycans make up about $2 \%$ of the stroma; the rest is soluble proteins $4 \%$, salts $1 \%$, and water $78 \%$. However, the proteoglycans are the molecules largely responsible for the swelling, hydration, and water retention in the cornea. ${ }^{1}$ Thus the stromal lamellae can be depicted as a Wiener mixed body of the first type: long cylindrical fibrils embedded in a ground substance of different refractive index.
The packing of lamellae are such that in each neighboring lamellae the collagen fibrils are run at various angles to one another. The lamellae are of different thicknesses and cells are scattered within the lamellae.

One could expect that such a structure would behave optically similarly to a swollen collagen film. Such films have been studied extensively by Kawai and his coworkers ${ }^{2,3,4}$ and by Chien and Chang ${ }^{5}$ regarding their light scattering properties. All these collagen films exhibit rod-like scattering, ${ }^{6}$ in which both the $I_{+}\left(H_{\mathrm{v}}\right)$ and the $I_{\|}\left(V_{\mathrm{v}}\right)$ patterns exhibit a four lobe type of scattering but the two modes are displaced $45^{\circ}$ to each other. This means that if $I_{+}$is of the $0-90^{\circ}+$-type the $I_{\|}$is of the $45^{\circ} \times$-type and vice versa. Theoretical models for these scattering patterns have been worked out including optically anisotropic rods of finite length but infinitely thin with random orientation in 2 or 3 dimensional space $\mathrm{s}^{6,2,3}$ or rods with finite length and width oriented in 3 dimensional space. $^{7-9}$

Attempts to draw an analogy between collagen films and the cornea have a twofold importance. 


\section{Kumbar and F. A. Bettelheim}

From the point of view of ophthalmology it has been proposed ${ }^{10}$ that suitably modified collagen films can act as a substitute for damaged corneas. If such transplants are feasible, the optical properties of the film must correspond to that of the cornea replaced. The second importance of the analogy lies in the fact that if the light-scattering patterns of cornea and collagen films are similar, we may learn about the structure of the cornea from the wealth of models proposed for collagen scattering.

Unfortunately, this is not the case. Lightscattering patterns of bovine ${ }^{11,12}$ cornea in the $I_{+}$mode show a + arrangement in which one set of lobes is more prominent than the other set normal to it. Furthermore, the $I_{\|}$patterns do not show a four lobe pattern at all, but an anisotropic pattern along the direction that was more intense in the $I_{+}$mode. Similar light scattering patterns were obtained on a few human corneas (unpublished results).

Rabbit ${ }^{13}$ and rat $^{14}$ corneas exhibit a cloverleaf arrangement with 5 maxima in the $I_{+}$mode. While the $I_{||}$mode of rabbit cornea ${ }^{13}$ is similar to that observed in bovine cornea, ${ }^{12}$ the $I_{\|}$mode of rat cornea ${ }^{14}$ is completely nondescript isotropic.

Most of the light scattered by bovine cornea occurs at small angles ${ }^{11,12}$ and we proposed that the scattering originates largely from the birefringence of aggregates of collagen fibrils. The birefringence is partly due to the preferential orientation of optically anisotropic collagen fibrils in the different parts of the cornea that is symmetrically distributed and partly from form birefringence. ${ }^{14}$ Similar considerations apply to rabbit ${ }^{13}$ and rat $^{14}$ cornea.

The fact that the scattering patterns of cornea were unlike those of collagen films indicate that a special model of rod-like structures must be designed to interpret the corneal scattering.

In the following we attempt to develop the light-scattering theory of such a model and by matching experimental and computed theoretical light-scattering patterns we shall interpret the corneal structure in terms of the parameters of the theory.

\section{THEORETICAL}

We start with the model of two-dimensional

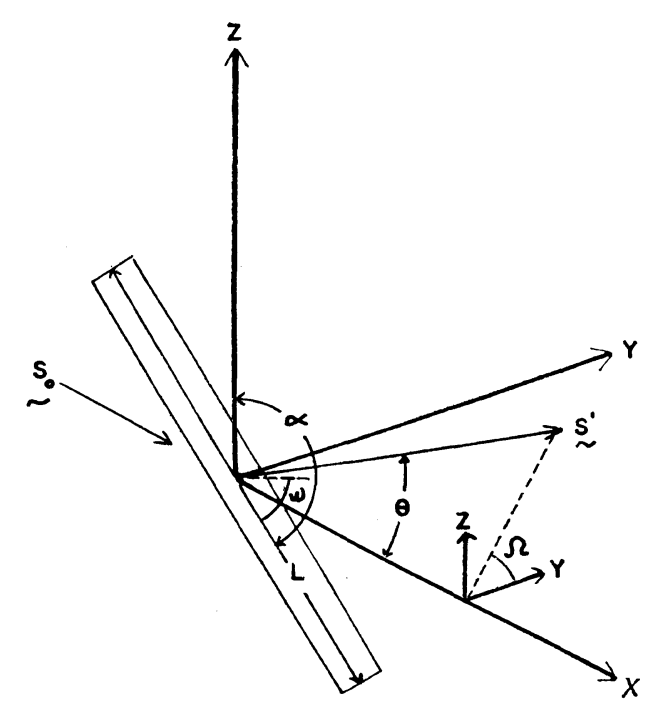

Figure 1. Model of an anisotropic rod: $Z$, the direction of polarization of the laser beam; $\mathbf{s}_{0}$, the incident beam; $\mathbf{s}^{\prime}$, the scattered beam. For the rest see next.

assembly of anistropic rods which was developed by Rhodes and Stein. ${ }^{6}$ Figure 1 shows the coordinate system used to relate the scattered beam to the length of the rod $L$, to the orientation of the $\operatorname{rod} \alpha$, and to the direction of maximum polarizability $\omega$ of the rod. The origin of the coordinate system is placed at the center of the rod. The rod is assumed to have infinitesimal thickness. The angle $\alpha$ measures the tilt of the rod as measured from the $z$ axis. The $\psi$ is the angle between the $x$ axis and the projection of the rod in the $x y$ plane. When the rod is restricted to lie in the $y z$ plane, that is, for the two-dimensional model, the angle $\psi$ is equal to $90^{\circ}$. The angle $\theta$ and $\Omega$ are the two angles that define the unit vector $s^{\prime}$ in the direction of the scattered radiation. The $\theta$ is the angle between the scattered ray and the incident ray unit vector $s_{0}$ along the $x$ axis. The $\Omega$ is the angle between the $s^{\prime}$ and the $y$ axis in the $y z$ plane and is measured from the $y$ axis. The angle $\omega$ is the direction of the maximum polarizability for the rod as measured from the long geometric axis of the rod and is confined to lie in the same plane as the angle $\alpha$.

In the following we make use of the derivations of Rhodes and Stein. The intensity 
Light Scattering from Nonrandom Anisotropic Rods

distribution of a two-dimensional nonrandom assembly of rods may be given by

$$
I=\iint p(\alpha, \phi) A^{2} \mathrm{~d} \alpha \mathrm{d} \psi
$$

where $P(\alpha, \psi)$ is the distribution function. When the assembly of rods is restricted to a twodimensional collection, the angle $\phi$ becomes $90^{\circ}$ and the distribution function $P(\alpha, \phi)$ reduces to $P(\alpha) . \quad A$ is the total amplitude of the scattered rays from the scattering elements within the rod, and is written as

$$
A=\rho_{0} \int(\boldsymbol{M} \cdot \boldsymbol{O}) \cos \frac{2 \pi}{\lambda}(\boldsymbol{r} \cdot \boldsymbol{s}) \mathrm{d} r
$$

In eq 2, $\rho_{0}$ is the scattering power of a rod per unit length per unit incident field strength when the rod is oriented at $\alpha=0^{\circ}$. The $O$ is a unit vector along the polarization direction of the analyzer. $\boldsymbol{O}=\boldsymbol{j}$ for horizontal scattering, and $\boldsymbol{O}=\boldsymbol{k}$ for vertical scattering. The $\boldsymbol{s}$ is the scattering vector, which is defined by

$$
\begin{aligned}
\boldsymbol{s}= & \left(\boldsymbol{s}_{0}-\boldsymbol{s}^{\prime}\right)=(1-\cos \theta) \boldsymbol{i}-(\sin \theta \cos \Omega) \boldsymbol{j} \\
& +(\sin \theta \sin \Omega) \boldsymbol{k}
\end{aligned}
$$

In the above equation, the $s_{0}=i$, the unit vector along the $x$ axis, and $s^{\prime}$ is the unit vector along the scattered ray:

$$
\boldsymbol{s}=\cos \theta \boldsymbol{i}+(\sin \theta \cos \Omega) \boldsymbol{j}+(\sin \theta \sin \Omega) \boldsymbol{k}
$$

Then the expressions for $I_{\|}$mode (polarizer and analyzer are parallel) and for $I_{+}$mode (polarizer and analyzer are at $90^{\circ}$ to each other) are obtained by substituting eq 2 and 3 into eq 1 . These equations are

and

$$
\begin{aligned}
I_{\|}= & {\rho_{0}}^{2} \iint_{0}^{\pi} P(\alpha, \psi)(\boldsymbol{M} \cdot \boldsymbol{k})^{2} \\
& \times\left[\int \cos \frac{2 \pi}{\lambda}(\boldsymbol{r} \cdot \boldsymbol{s}) \mathrm{d} \boldsymbol{r}\right]^{2} \mathrm{~d} \alpha \mathrm{d} \psi
\end{aligned}
$$

$$
\begin{aligned}
I_{+}= & \rho_{0}{ }^{2} \iint_{0}^{\pi} P(\alpha, \psi)(\boldsymbol{M} \cdot \boldsymbol{j})^{2} \\
& \times\left[\int \cos \frac{2 \pi}{\lambda}(\boldsymbol{r} \cdot \boldsymbol{s}) \mathrm{d} \boldsymbol{r}\right]^{2} \mathrm{~d} \alpha \mathrm{d} \psi
\end{aligned}
$$

The total induced dipole moment $M$ is expressed by the following equation:

$$
\boldsymbol{M}=E_{0}\left[\delta(\cos \alpha)(\boldsymbol{r} / \boldsymbol{r})+b_{\mathrm{t}} \boldsymbol{k}\right]
$$

The $\delta$ is the anisotropy, which is assumed to be

$$
\delta=b_{1}-b_{\mathrm{t}}
$$

The $b_{1}$ and $b_{\mathrm{t}}$ are defined as

$$
\begin{aligned}
& b_{1}=b_{1}{ }^{\prime}-b_{\mathrm{s}} \\
& b_{\mathrm{t}}=b_{\mathrm{t}}{ }^{\prime}-b_{\mathrm{s}}
\end{aligned}
$$

where $b_{1}{ }^{\prime}$ is the longitudinal polarizability of the rod, along the direction of its maximum polarizability, $b_{t}{ }^{\prime}$ is the polarizability normal to $b_{1}{ }^{\prime}$ and $b_{\mathrm{s}}$ is the polarizability of the surroundings. The equation for $r$ is

$$
\boldsymbol{r}=r[(\sin \alpha \cos \psi) \boldsymbol{i}+(\sin \alpha \sin \psi) \boldsymbol{j}+(\cos \alpha) \boldsymbol{k}]
$$

By substituting eq 3 and 7 into eq 5 and 6 , and evaluating the integral over $\mathrm{d} r$, the final expressions for $I_{\|}$and $I_{+}$for two-dimensional rods are:

$$
\begin{aligned}
I_{\|}= & \left(\rho_{0} E_{0} L\right)^{2} \int_{0}^{\pi} \mathrm{d} \alpha^{\prime} P\left(\alpha^{\prime}\right)\left(\delta \cos ^{2} \alpha^{\prime}+b_{\mathrm{t}}\right)^{2} \\
& \times\left[\frac{\sin (a x)}{a x}\right]^{2}
\end{aligned}
$$

and

$$
\begin{aligned}
I_{+}= & \left(\rho_{0} E_{0} L\right)^{2} \int_{0}^{\pi} \mathrm{d} \alpha^{\prime} P\left(\alpha^{\prime}\right) \delta^{2} \sin ^{2} \alpha^{\prime} \cos ^{2} \alpha^{\prime} \\
& \times\left[\frac{\sin (a x)}{a x}\right]^{2}
\end{aligned}
$$

where

and

$$
\begin{gathered}
a=-\sin \left(\alpha^{\prime}+\Omega\right) \sin \theta \\
x=\pi L / \lambda
\end{gathered}
$$

$$
\alpha^{\prime}=\alpha+\omega
$$

The $\lambda$ is the wavelength of the radiation. The $P\left(\alpha^{\prime}\right)=1$ for a random distribution and $P\left(\alpha^{\prime}\right) \neq 1$ nonrandom distribution.

Unlike Rhodes and Stein, we have assumed that the optical anisotropy arises due to the presence of intrinsic and form birefringence. Thus, the $\delta$ which measures the anisotropy is the sum of the two terms,

$$
\delta=\delta^{\prime}+B
$$

where $\delta^{\prime}$ is a measure of the intrinsic birefringence and $B$ is a measure of form birefringence.

The reason for separating the two contributions to birefringence lies in the fact that during the small unidirectional stresses the intrinsic birefringence changes with orientation while the form birefringence may stay constant. ${ }^{16}$ On the other hand, when migration of water from 
specific locus to locus rather than lamellar orientation occurs during a stress or when the refractive index of the embedding medium changes, the form birefringence changes without effecting in intrinsic birefringence. The latter situation definitely arises when a large amount of scattering (clouding) occurs during corneal swelling. ${ }^{12}$ We assume that both $B$ and $\delta^{\prime}$ are constants since the average shape and optical anisotropy of the individual collagen fibers are the same and only their average preferential orientation changes along the topography of the cornea. Thus, only $b_{\mathrm{t}}$ and $P\left(\alpha^{\prime}\right)$ are dependent on $\alpha$.

The assumption of the constancy of $\delta^{\prime}$ is justified as a first approximation since the local optic axis of the cornea is an operative optic axis as discussed below. Replacing $\delta$ in eq 12 and 13 and rearranging, the equations for $I_{\|}$and $I_{+}$take the following form:

$$
\begin{aligned}
I_{\|}= & \left(\rho_{0} E_{0} L\right)^{2} \int_{0}^{\pi} \mathrm{d} \alpha^{\prime} P\left(\alpha^{\prime}\right)\left(\delta^{\prime} \cos ^{2} \alpha^{\prime}+b_{\mathrm{t}}\right)^{2} \\
& \times\left[\frac{\sin (a x)}{a x}\right]^{2}+\left(\rho_{0} E_{0} L\right)^{2} \int_{0}^{\pi} \mathrm{d} \alpha^{\prime} P\left(\alpha^{\prime}\right) \\
& \times\left[B \cos ^{2} \alpha^{\prime}\left(B \cos ^{2} \alpha^{\prime}+2 \delta^{\prime} \cos ^{2} \alpha^{\prime}+2 b_{\mathrm{t}}\right]\right. \\
& \times\left[\frac{\sin (a x)}{a x}\right]^{2}
\end{aligned}
$$

and

$$
\begin{aligned}
I_{+}= & \left(\rho_{0} E_{0} L\right)^{2} \int_{0}^{\pi} \mathrm{d} \alpha^{\prime} P\left(\alpha^{\prime}\right) \delta^{\prime 2} \sin ^{2} \alpha^{\prime} \cos ^{2} \alpha^{\prime} \\
& \times\left[\frac{\sin (a x)}{a x}\right]^{2}+\left(\rho_{0} E_{0} L\right)^{2} \int_{0}^{\pi} \mathrm{d} \alpha^{\prime} P\left(\alpha^{\prime}\right) \\
& \times\left[B\left(2 \delta^{\prime}+B\right)\right] \sin ^{2} \alpha^{\prime} \cos ^{2} \alpha^{\prime}\left[\frac{\sin (a x)}{a x}\right]^{2}
\end{aligned}
$$

In each of the above equations the second term exists due to the inclusion of the form birefringence. If there is no contribution from the form birefringence, that is, for $B=0$, eq 18 and 19 reduce to eq 12 and 13 .

The second innovation in our model is the introduction of a distribution function that can have its maximum at any $\alpha$ angle. The reason for this step lies in the experimental patterns, ${ }^{12}$ which showed that the collagen fibrils took up an averaged specific orientation in the different parts of the cornea that were at some specific $\alpha$ angle to the direction of the polarization of the laser beam.

The distribution function selected is a zeroorder logarithmic distribution function (ZOLD), ${ }^{17}$ which has the form

$$
\begin{aligned}
P\left(\alpha^{\prime}\right)= & \left\{\exp \left[-\frac{\left(\log \alpha^{\prime}-\log \alpha_{\mathrm{M}}\right)^{2}}{2 \sigma_{0}{ }^{2}}\right]\right\} \\
& \div\left\{(2 \pi)^{1 / 2} \sigma_{0} \alpha_{\mathrm{M}}\left[\exp \left(\sigma_{0}{ }^{2} / 2\right]\right\}\right.
\end{aligned}
$$

In eq $20, \alpha$ is the angle between the long axis of the rod and the $Z$ coordinate axis (polarization of incident laser beam). $\alpha_{M}$ is the value of $\alpha$ at the maximum of the distribution function and $\sigma_{0}$ is a measure of the width and skewness of the distribution function. In our treatment the distribution function is not normalized. This has no effect upon the computed scattering pattern since the normalization constant would be part of the multiplier in front of the integrand in eq 18 and 19 and we are dealing with relative intensities in any case.

The $P\left(\alpha^{\prime}\right)$ is an assymmetric distribution and thus, in principle, would not satisfy the physical requirement that two rods oriented at $\alpha$ and at $\alpha+\pi$ angle should be indistinguishable. However, the nature of this distribution function is such that it remains fairly constant between $\alpha=0$ and $\alpha=2 \pi$ for particular values of $x, \omega$, $\sigma_{0}, \alpha_{\mathrm{M}}, \delta$ and $B$. Therefore as a approximation one can replace $\int_{0}^{2 \pi} \mathrm{d} \alpha^{\prime}$ with $2 \int_{0}^{\pi} \mathrm{d} \alpha^{\prime}$ and thus the physical requirement of twofold symmetry in the scattering patterns is preserved.

Furthermore, in the treatment we assumed both positive and negative values of $\omega$ are equally probable in eq 16 .

The parameter $b_{t}$ is a function of the tangential polarizability of the rod. If the rods have specific average orientation, $b_{\mathrm{t}}$ itself will be a function of $\alpha$. The reason for this assumption is as follows: Coulombre and Coulombre ${ }^{18}$ have proved that the optical axis of a cornea is more or less normal to the surface of the cornea. The laser beam normal to the surface sees the projection of the local optical axis into the plane of the cornea. This projection is then operative local optical axis. We take it that this is an intrinsic property of the cornea and it is a constant at a set location. The twodimensional rod model then will have two 
mutually perpendicular components of this operative local axis, $b_{1}{ }^{\prime}$ and $b_{t}{ }^{\prime}$, and their values will depend on the orientation of the rod. Thus, in contrtst to the Rhodes and Stein model, our $b_{\mathrm{t}}$ is not an intrinsic property of the rod. In evaluating the angular dependence of $b_{t}$, we selected a semiempirical method. Since the experimental patterns had the same angular dependence $^{12}$ we took the second derivatives with respect to $\alpha^{\prime}$ of eq 18 and 19 and set them equal to zero.

The $b_{t}$ thus obtained had the form

$$
b_{t}=\frac{3 \delta \cos ^{4} \alpha^{\prime}}{\left(4 \sin ^{2} \alpha^{\prime}+\cos ^{2} \alpha^{\prime}\right)}
$$

Using eq $18,19,20$, and 21 , we generated theoretical light-scattering patterns in a CDC 3600 computer by feeding in different parameters of $\omega, x, \sigma_{0}, \alpha_{M}, \delta^{\prime}$, and $B$. The intensities of $I_{\|}$ and $I_{+}$modes were obtained as a function of two scattering angles $\theta$ and $\Omega$.

\section{RESULTS AND DISCUSSION}

In evaluating the effect of different parameters in our model we used constant terms for the two kinds of birefringence. These were representative values obtained experimentally ${ }^{1,15}$ although as was proved the birefringence varies with the topographic location of the scattering units in the cornea. ${ }^{15}$ We selected values of $4 \times 10^{-3}$ for form birefringence, $B$, and $2 \times 10^{-3}$ for intrinsic birefringence, since experiments indicated $^{1,15}$ that the form birefringence is about $2 / 3$ of the total birefringence in a cornea.

Figure 2 depicts the effect of the length of the
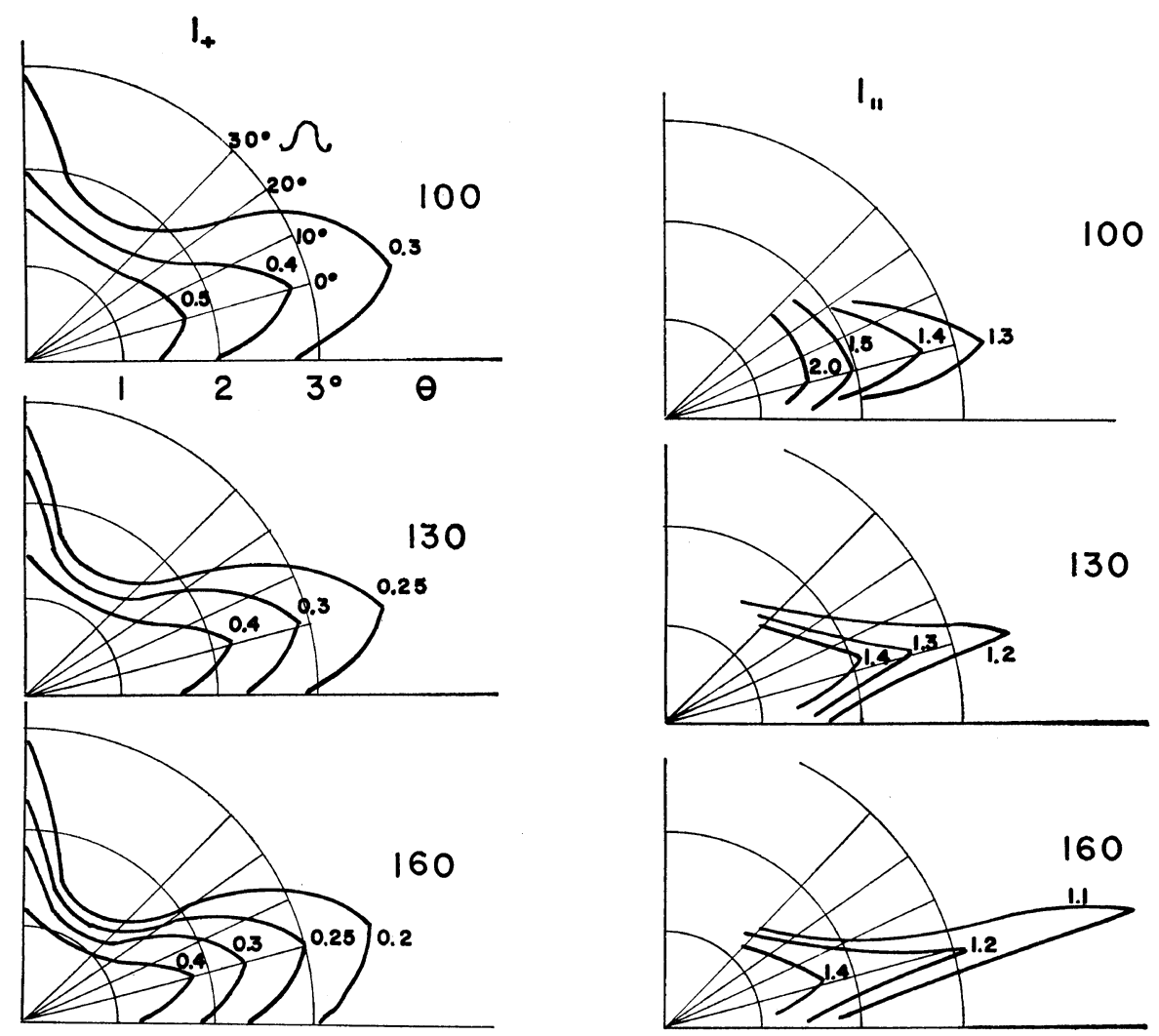

Figure 2. Computer-simulated $I_{\|}$and $I_{+}$patterns as a function of length of the rod, $x, 100,130$, 160: $B, 4 \times 10^{-3} ; \delta^{\prime}, 2 \times 10^{-3} ; \sigma, 2.0 ; \alpha_{M}, 120^{\circ} ; \omega^{\circ}, 60^{\circ}$. On the first drawing the coordinates of the scattering patterns are given in numerical terms both for $\theta$ and $\Omega$. In subsequent drawings the same numerical values are applicable. 


\section{Kumbar and F. A. Bettelheim}

rod on the light-scattering patterns. We selected values of 100,130 , and 160 for $x=\pi L / \lambda$, which is equivalent to 20,26 , and $32 \mu$ lengths of a rod; here $\lambda$ is the wavelength of the $\mathrm{He}-\mathrm{Ne}$ laser, $632.8 \mathrm{~nm}$. Two effects can be observed on the $I_{\|}$patterns: as the length of the rod increases the width as well as the length of the geometrically anisotropic pattern decreases. This means that as the length of the rod increases, a set intensity of scattered light shifts to lower $\theta$ angles. The $\Omega$ angle is also effected in the same way but to a much smaller extent than the $\theta$ angle. The corresponding intensities of $I_{+}$patterns at set $\theta$ and $\Omega$ angles are about $1 / 4$ of the $I_{\|}$patterns. The length of the rod has a similar although somewhat more pronounced effect on the $I_{+}$patterns relative to the effect on the $I_{\|}$. The larger the length of the rod, the smaller the $\theta$ angle at which the same intensity isochore will appear. The $\Omega$ angle is
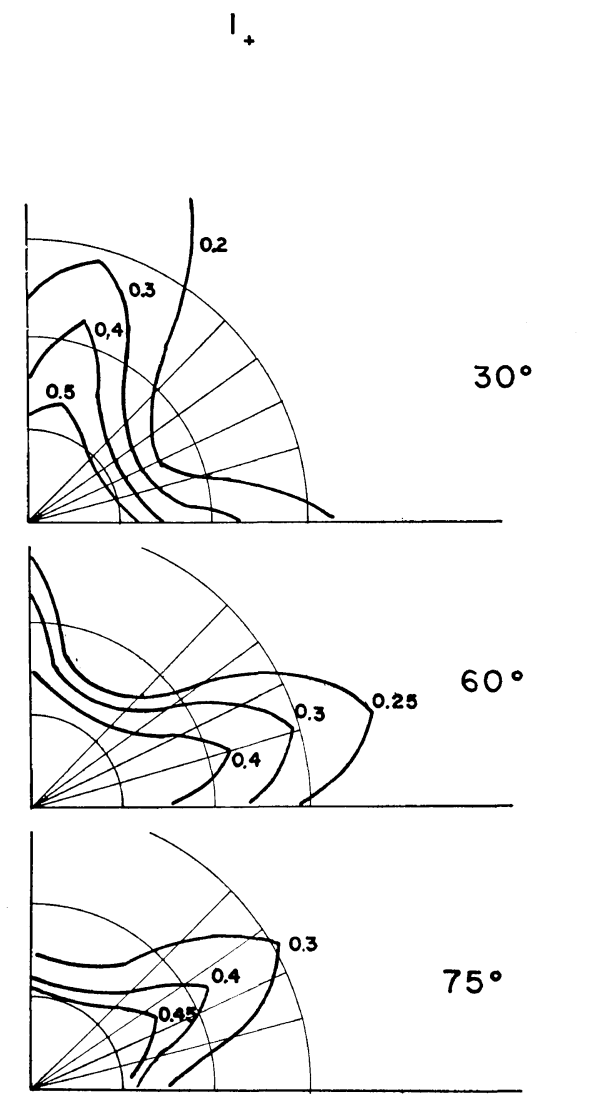

$30^{\circ}$
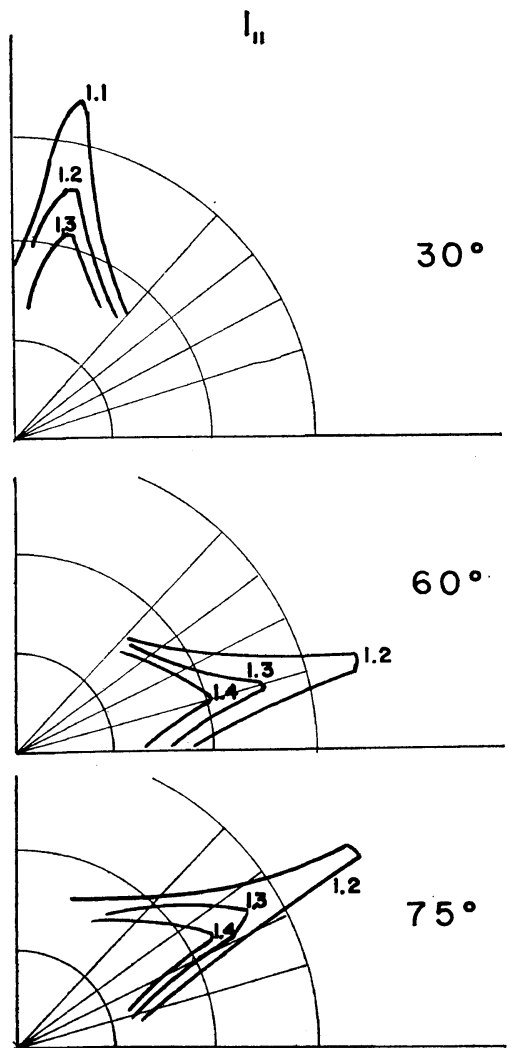

Figure 3. Computer-simulated $I_{\|}$and $I_{+}$patterns as a function of $\omega$ angle, $\omega, 30,60,75^{\circ}: B, 4 \times 10^{-3}$; $\delta^{\prime}, 2 \times 10^{-3} ; x, 130 ; \sigma, 2.0 ; \alpha_{\mathrm{M}}, 120^{\circ}$. 
is however, inherent in the model and thus nothing unexpected. The intensity of the $I_{+}$ pattern increases with incresing $\omega$ angle, but only very slightly. Thus, changing $\omega$ from $30^{\circ}$ to $60^{\circ}$ moves the 0.3 intensity isochore to an only slightly higher $\theta$ value and a further increase to $\omega=75^{\circ}$ affects the position of the 0.3 intensity isochore again only slightly. The most drastic effect of $\omega$ is on the intensity of the weaker set of the + lobes of the pattern. As $\omega$ increases the intensity of this set of lobes decreases; thus the same intensity isochore appears at lower $\theta$ values.

The $\omega$ does not affect the intensity ratio of the $I_{\|} / I_{+}$patterns, which stays at a value of 4 .

In Figure 4, the effect of the width parameter of the distribution function, $\sigma$, is presented. The $\sigma$ chiefly influences the intensity of the scattering patterns. Changing $\sigma$ from 1 to 2 , the intensity of the $I_{\|}$pattern at a set $\theta$ value decreases 10-fold; going to $\sigma=2.5$ another 4-fold decrease in intensity is calculated. The shape and the orientation of the $I_{\|}$pattern is not affected by $\sigma$. In the $I_{+}$pattern, the decrease in intensity is somewhat less: when $\sigma=1.0$ changed to $\sigma=2.0$, the result was a 8 -fold decrease in intensity; giving $\sigma$ the value of 2.5, a 3.5-fold decrease in intensity was effected. The $I_{\|} / I_{+}$ratio of 4 was not affected by $\sigma$.

Figure 5, describes the influence of the $\alpha_{\mathrm{M}}$, i.e., the average orientation of the rod, on the light-scattering patterns. The main effect of $\alpha_{M}$ is on the intensities of both $I_{\|}$and $I_{+}$patterns. As $\alpha_{M}$ increases the intensity of the scattering pattern decreases, in other words, the same intensity isochore appears at smaller $\theta$ values. This can be seen on both $I_{\|}$and $I_{+}$patterns. What is unique for $\alpha_{\mathrm{M}}$ is that it also changes the $I_{\|} / I_{+}$ratios at set $\theta$ values. This effect seems quite complex. For one, the ratio changes with $\theta$ angles within one set of $I_{\|}$and $I_{+}$pairs. For another, going from $\alpha_{\mathrm{M}} 120$ to $135^{\circ}$ affects
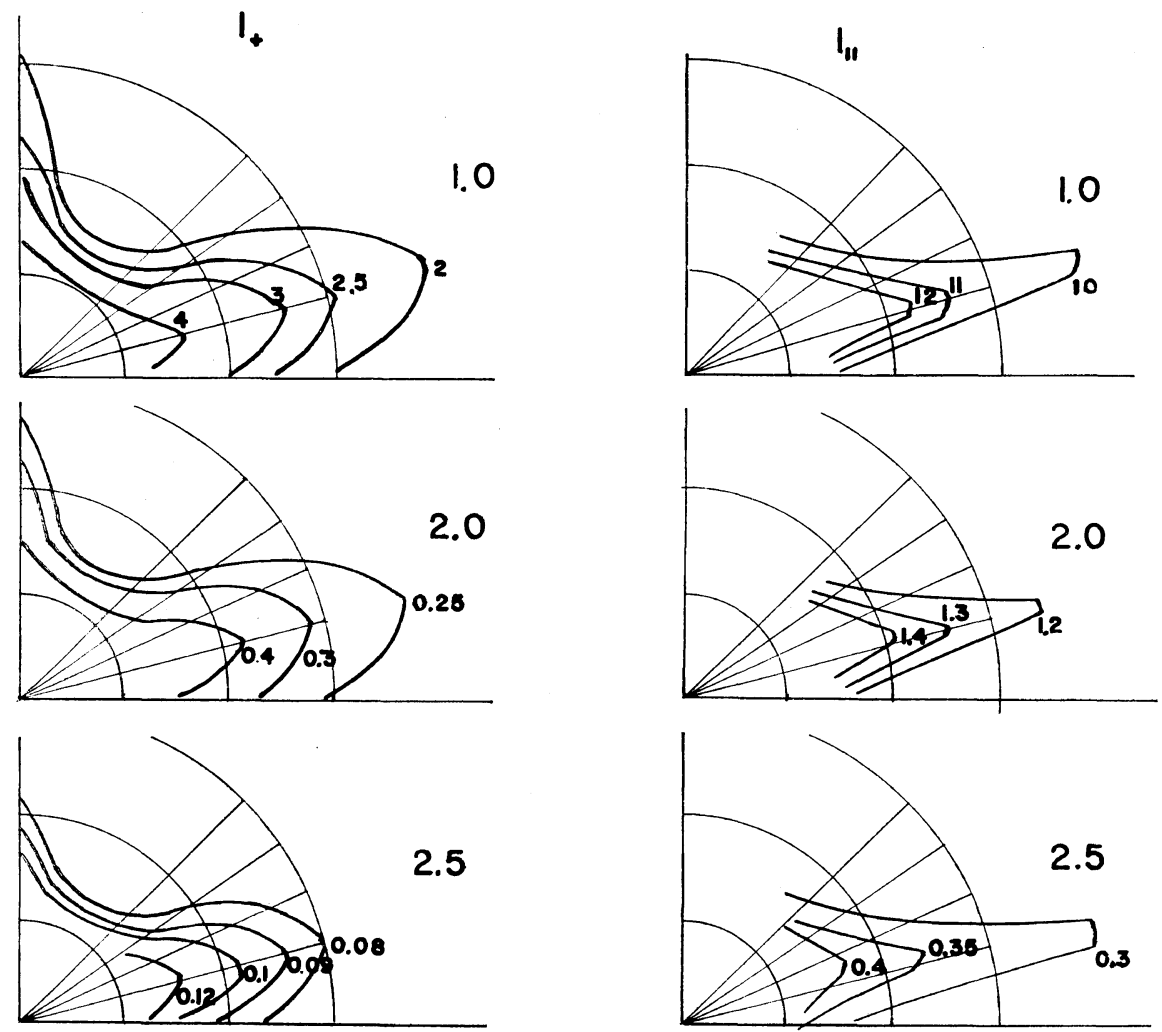

Figure 4. Computer-simulated $I_{\|}$and $I_{+}$patterns as a function of width of the distribution function, $\sigma, 1.0,2.0,2.5: \quad B, 4 \times 10^{-3} ; \delta^{\prime}, 2 \times 10^{-3} ; \omega, 60^{\circ} ; x, 130 ; \alpha_{\mathrm{M}}, 120^{\circ}$. 

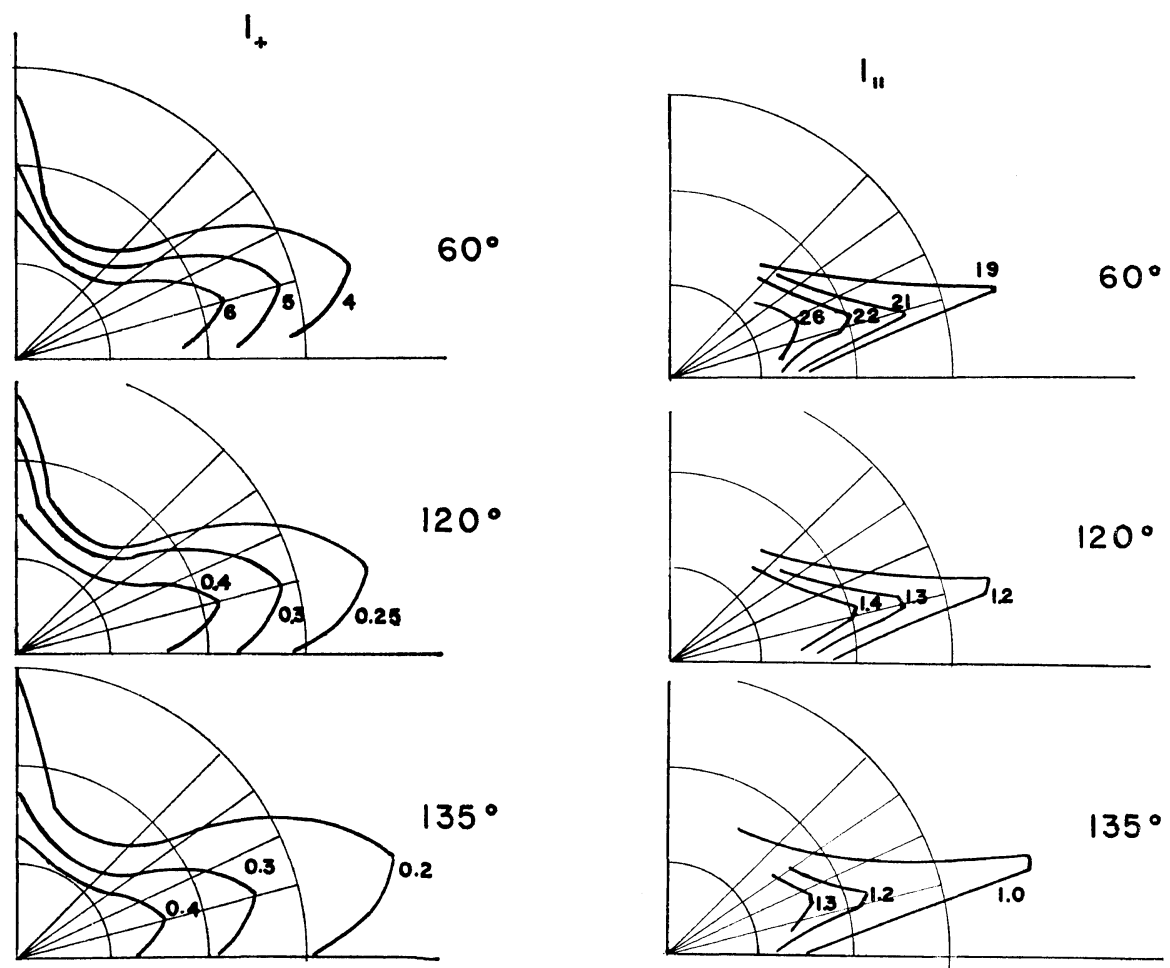

Figure 5. Computer-simulated $I_{\|}$and $I_{+}$patterns as a function of the average orientation of the scattering rod, $\alpha_{\mathrm{M}}, 60,120$, and $135^{\circ}: B, 4 \times 10^{-3} ; \delta^{\prime} ; 2 \times 10^{-3} ; \omega, 60^{\circ} ; x, 130 ; \sigma, 2.0$.

the ratios at a set $\theta$ more than moving $\alpha_{M}$ from 60 to $120^{\circ}$.

After elucidating the effect of individual parameters on the light-scattering patterns calculated from our model the final equation was raised: "Can one match the experimentally obtained patterns with those of computer-simulated patterns?" To answer this point we selected a set of $I_{+}$and $I_{\|}$experimental patterns obtained on bovine cornea. ${ }^{12}$ These are reproduced in Figure 6, and the densitometric tracing of the photographs are given in Figure 7 , together with computer-simulated patterns that are close though not identical with the experimental patterns.

Although qualitatively the experimental and theoretical isochores correspond, the intensity ratios of $I_{\|} / I_{+}$are comparable only at small $\theta$ angles, $2^{\circ}$ and below, and the wider the angle the more discrepancy is found between the real and the model system. This is, however, not unusual and similar attempts in reconciling ex- perimental data with model systems have been encountered in the literature. ${ }^{2,19}$ Model fitters are usually satisfied if the computer simulated patterns have the same angular distribution as the experimental patterns, without worrying much about discrepancies in intensities.

Keeping this difficulty in mind, then, one can say that the numerical parameter obtained from such matching have reasonable good physical meaning. The length of the rod $1.26 \mu$, as a scattering center is reasonable. Small angle scatters are all in the 10 and above micron range. Laser diffraction patterns of bovine lenses at such angles also yielded dimensions between $10-20 \mu$ that can be confirmed by electron microscopy. ${ }^{20}$ The width of the distribution function of rod orientation $\sigma=2.0$ is also reasonably wide, indicating that the preferential orientation is really superimposed upon a random orientation. Similar conclusions were reached earlier. ${ }^{11,12}$

The most interesting result is that the main polarizability axis does not coincide with the 


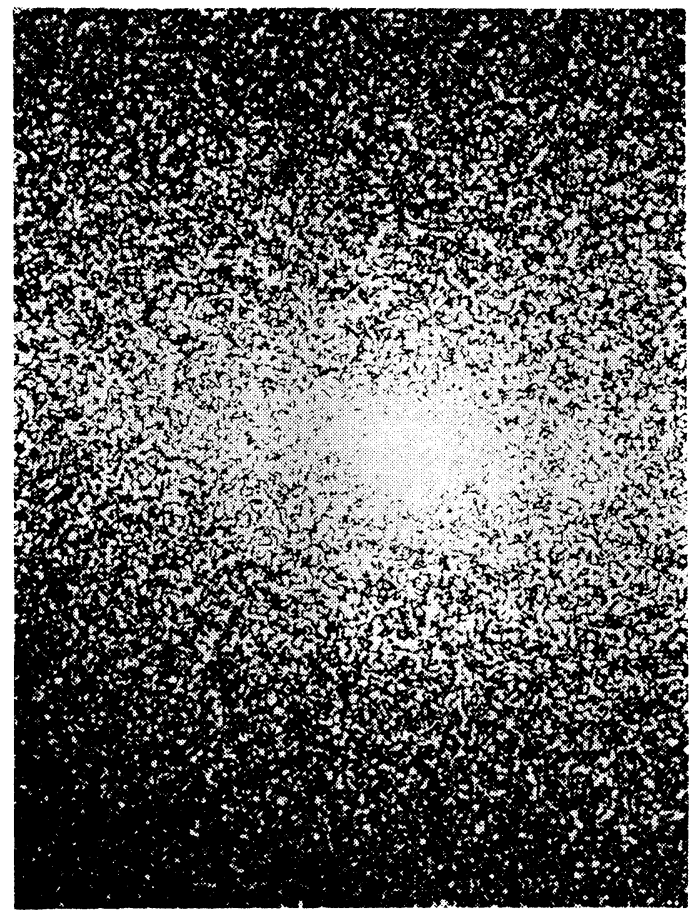

(a)

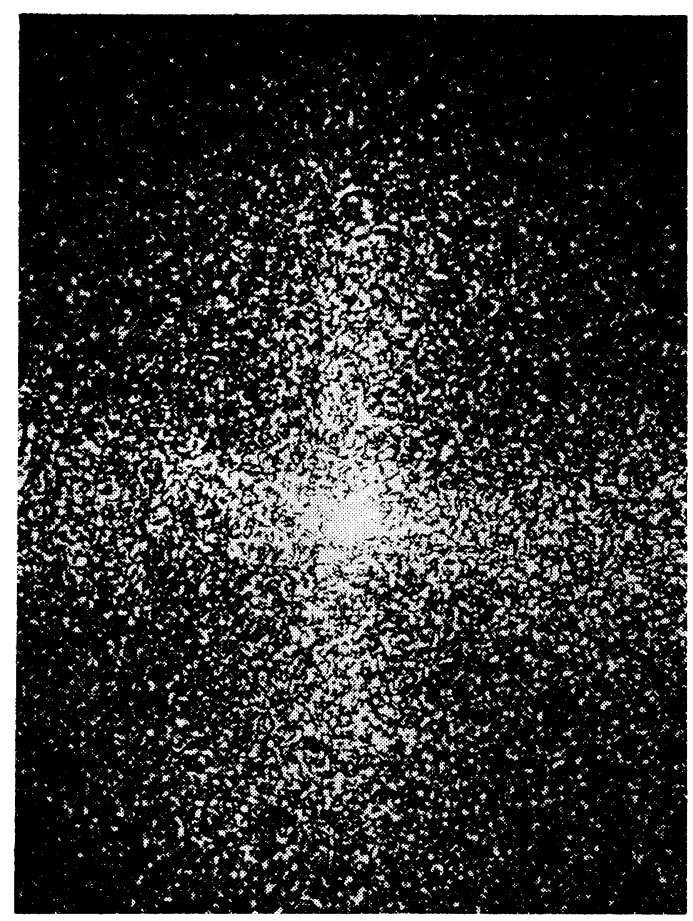

(b)

Figure 6. Experimental $I_{\|}$and $I_{+}$patterns of bovine cornea.
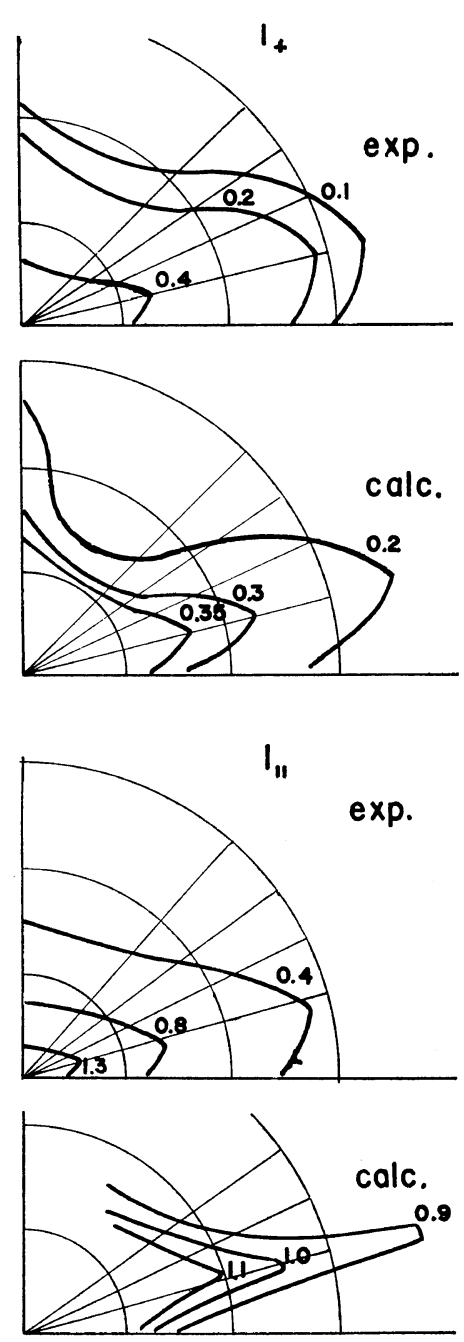

Figure 7. $I_{\|}$and $I_{+}$patterns of densitometric scan of Figure 6, and their matching computer-simulated patterns: $x, 130 ; B, 0.004 ; \delta^{\prime}, 0.002 ; \omega, 60^{\circ} ; \sigma$, $2.0 ; \alpha_{\mathrm{M}}, 150^{\circ}$.

geometric long axis of our rod scatterer. The $\omega$ angle obtained is $60^{\circ}$. This is in close agreement with the results of Moritani, et al., ${ }^{2}$ and also with that of Chien and $\mathrm{Chang}^{5}$ on collagen films. The former authors obtained a value between $50-70^{\circ}$ and the latter $50^{\circ}$. Since the scattering unit is large, it cannot clearly be identified with a definite structural unit of collagen. Supermolecular structures are involved, namely the tropocollagen molecules are built in protofibrils and subsequently in fibrils and finally in 


\section{Kumbar and F. A. Bettelheim}

fibers. Although the collagen structure at the molecular level is well known, ${ }^{23}$ the superstructures of collagen fiber in vivo are not well defined as yet. Therefore, it is difficult to tell whether the $\omega=60^{\circ}$ represents the optical anisotropy of the collagen molecules or the diagonal arrangement of the protofibrils and fibrils within our rod scatterer. Evidence for the latter structure was found by Bouteille and Pease, ${ }^{22}$ who proposed that tropocollagen aggregates of 3035-nm diameter wind in a helical pattern within the collagen fibrils with a gyre length slightly exceeding $1 \mu \mathrm{m}$.

The finding that the average orientation of the rod scatterer in the cornea is at $150^{\circ}$ angle to the polarization of the laser beam is not surprising at all. As a matter of fact this is a direct consequence of our experimental technique. In the laser scattering of a cornea, we wanted to minimize the secondary effect of birefringence on the experimental patterns. ${ }^{23-25}$ Therefore, we aligned the polarization of the laser beam perpendicular to the local optical axis. Thus $\alpha_{\mathrm{M}}=150^{\circ}$ is actually $\omega+90^{\circ}$, where $\omega=60^{\circ}$.

In the final analysis the model of a twodimensional nonrandom assembly of optically anisotropic rods can account well for the lightscattering patterns obtained on human and bovine cornea at small angles and the physical parameters yielded by this model have definite significance in the analysis of the molecular superstructures. At the same time, we must bear in mind that this model is a simplified version of the real situation. (Further modifications may be introduced to account for the corneal light-scattering patterns of rats and rabbits.) The model assumes that the rods are independent scatterers and there is no correlation in the orientation. If correlation exists, the angular dependence of the scattering intensity will depend very much on the nature of the correlation function. Prud'homme and $\mathrm{Stein}^{26}$ have worked out the mathematics for a few simple cases of correlations. Even in such simple cases, it is very hard to separate the correlation parameters from the rod parameters. However, such a correlation may be important at small angles (below $1.5^{\circ}$ ) where theory and experiment deviate most. ${ }^{4,9}$
The advantage of our simplified model is that it yields rod parameters. In essence, we deal with the correlation of rod orientation by assuming that those rods which have perfectly parallel alignment to each other in the lamellae will cause destructive interference. ${ }^{27}$ Therefore, they do not contribute to the angular distribution of the intensity of the scattered light. Since 95\% of the light passes through the normal cornea, the majority of the rods belong to this group. The remaining rods are then taken as independent scatterers and their contribution is summed up in the experimental light-scattering pattern.

Acknowledgment. This research was supported by a National Eye Institute, Grant EY 00501-05, P.H.S. We would like to thank Prof. R. S. Stein for providing a preprint of his manuscript and for the other helpful suggestions.

\section{REFERENCES}

1. D. M. Maurice in "The Eye" H. Davson, Ed., 2nd ed., Vol. 1, Academic Press, New York, N.Y., Chapter 7 (1969).

2. M. Moritani, N. Hayashi, A. Utsuo, and H. Kawai, Polymer J., 2, 74 (1971).

3. Y. Murakami, N. Hayashi, T. Hashimoto, and H. Kawai, ibid., 4, 452 (1973).

4. T. Hashimoto, Y. Murakami, N. Hayashi. and H. Kawai, ibid., 6, 132 (1974).

5. J. C. W. Chien and E. P. Chang, Macromolecules, 5, 610 (1972).

6. M. B. Rhodes and R. S. Stein, J. Polym. Sci., Part A-2, 7, 1539 (1969).

7. J. J. Van Aartsen, Eur. Polym. J., 6, 1095, (1972).

8. N. Hayashi and H. Kawai, Polymer J., 3, 140 (1972).

9. M. Matsuo, S. Nomura, T. Hashimoto, and H. Kawai, ibid., 6, 151 (1974).

10. M. W. Dunn, T. Nishihara, K. H. Stenzel, A. W. Branwood, and A. L. Rubin, Science, 157, 1329 (1967).

11. F. A. Bettelheim and M. J. Vinciguerra, Biochim. Biophys. Acta, 177, 259 (1969).

12. F. A. Bettelheim and D. Kaplan, ibid., 313, 268 (1973).

13. E. P. Chang, D. A. Keedy, and J. C. W. Chien, ibid., 343, 615 (1974).

14. F. A. Bettelheim and T. J. Y. Wang, Exp. Eye Res., 19, 511 (1974).

15. D. Kaplan and F. A. Bettelheim, ibid., 13, 310 (1972). 


\section{Light Scattering from Nonrandom Anisotropic Rods}

16. J. Cejtlin, M. J. Vinciguerra, and F. A. Bettelheim, Biochim. Biophys. Acta, 237, 530 (1971).

17. M. Kerker, "Scattering of Light," Academic Press, New York, N.Y., 1969, p 356.

18. A. J. Coulombre and J. L. Coulombre, in "The Structure of the Eye," Ed. G. Smelser, Academic Press, New York, N.Y., 1961, p 405.

19. R. J. Samuels, J. Polym. Sci., Part A-2, 9, 2165 (1971).

20. F. A. Bettelheim and M. J. Vinciguerra, Ann. N.Y. Acad. Sci., 172, 427 (1971).

21. G. N. Ramachandran, "Chemistry of Collagen,"
Academic Press, New York, N.Y., 1967.

22. M. Bouteille and D. C. Pease, J. Ultrastructure Res., 35, 314 (1971).

23. R. S. Stein, P. F. Erhardt, and W. Chu, J. Polym. Sci., Part A-2, 7, 271 (1969).

24. W. Chu and R. S. Stein, ibid., Part A-2, 8, 489 (1970).

25. N. Hayashi, Y. Murakami, M. Moritani, T. Hashimoto, and H. Kawai, Polymer J., 4, 560 (1973).

26. R. E. Prud'homme and R. S. Stein. J. Polym. Sci. (Polym. Phys.), 12, 1805 (1974).

27. D. M. Maurice, J. Physiol., 136, 263 (1957). 